\title{
Levels of cytokines in the aqueous humor guided treatment of refractory macular edema in adult-onset coats' disease
}

\author{
Yewei Wang ${ }^{1+}$, Hua Fan ${ }^{2 \dagger}$, Ke Gao ${ }^{1}$, Wei $\mathrm{He}^{3^{*}}$ and Yong Tao ${ }^{4^{*}}$ (D)
}

\begin{abstract}
Background: Two cases with refractory macular edema secondary to adult-onset Coats' disease underwent unsatisfactory treatment by intravitreal injections of anti-vascular endothelial growth factor (VEGF) drugs and retinal photocoagulation.

Case presentation: The authors highlight the guiding effect of the measurement of cytokines in the aqueous humor for the treatment of adult-onset Coats' disease with refractory macular edema. In the two cases, typical Coats' disease changes, including telangiectasis, subretinal exudation and macular edema were observed. Initial treatment consisted of intravitreal anti-VEGF drugs and retinal laser photocoagulation; however, the response was poor. Then, the aqueous humor was acquired and the cytokine concentrations were measured (Flow Cytometry Analysis, Beijing Giantmed Medical Diagnostics Lab). When the cytokine levels were tested every time there would be quality control, with a fixed concentration of cytokines samples to detect before the results reported. A low level of VEGF and a high level of inflammatory cytokines were found. Then, treatment was switched to intravitreal injection of dexamethasone implant (Ozurdex ${ }^{\circledR}$ ) (Allergan, Inc., Irvine, Calif., USA), which resulted in resolution of the refractory macular edema and improvement of visual acuity in both cases.
\end{abstract}

Conclusions: For refractory macular edema secondary to adult-onset Coats' disease, measurement of the levels of VEGF and inflammatory cytokines can help clinic doctors precisely investigate the molecular mechanism of macular edema and thereby find a suitable treatment.

Keywords: Macular edema, Adult-onset coats' disease, Case report

\section{Background}

Coats' disease is an idiopathic, nonhereditary retinal vascular disorder characterized by retinal telangiectasis, subretinal lipid exudation, macular edema and capillary nonperfusion areas. It is typically seen in childhood; adult onset is less common. Macular edema is one of

\footnotetext{
*Correspondence: hewei0111@163.com; drtaoyong@163.com

${ }^{\dagger}$ Yewei Wang and Hua Fan contributed equally to this work.

${ }^{3}$ Department of Ophthalmology, He Eye Hospital, He University, No.128,

North Huanghe Street, Shenyang 110034, China

${ }^{4}$ Department of Ophthalmology, Beijing Chaoyang Hospital, Capital Medical University, No. 8, South Road of Worker's Stadium, Chaoyang District, Beijing 100020, China

Full list of author information is available at the end of the article
}

the major causes of vision impairment related to adultonset Coats's disease.

A conclusive etiology of Coats' disease has not yet been determined. Several studies have reported increased VEGF levels in the aqueous humor of patients with Coats' disease [1, 2]. Several case reports have shown that intraocular injection of anti-VEGF combined with laser photocoagulation was effective for adult-onset Coats' disease [3, 4]. However, treatment of adult-onset Coats' disease has not been sufficiently investigated, and there have been poor refractory macular edema responses to anti-VEGF treatment. In this paper, we report two cases of adult-onset Coats' disease with a low level

C C The Author(s). 2020 Open Access This article is licensed under a Creative Commons Attribution 4.0 International License, which permits use, sharing, adaptation, distribution and reproduction in any medium or format, as long as you give appropriate credit to the original author(s) and the source, provide a link to the Creative Commons licence, and indicate if changes were made. The images or other third party material in this article are included in the article's Creative Commons licence, unless indicated otherwise in a credit line to the material. If material is not included in the article's Creative Commons licence and your intended use is not permitted by statutory regulation or exceeds the permitted use, you will need to obtain permission directly from the copyright holder. To view a copy of this licence, visit http://creativecommons.org/licenses/by/4.0/. The Creative Commons Public Domain Dedication waiver (http://creativecommons.org/publicdomain/zero/1.0/) applies to the data made available in this article, unless otherwise stated in a credit line to the data. 
of VEGF and a high level of inflammatory cytokines in the aqueous humor that responded more favorably to intravitreal injection of $\mathrm{Ozurdex}^{\circ}$ compared to anti-VEGF drugs.

\section{Case presentation}

\section{Case 1}

A 25-year-old man, without previous medical history, was presented to our eye clinic with decreased visual acuity in the left eye for 3 months. The best corrected visual acuity (BCVA) was 0.3 in the left eye and 1.0 in the right eye, with normal intraocular pressures. A massive intraretinal lipid accumulation with overlying vascular telangiectasis and hemorrhages was observed in the left eye (Figs. 1 and 2a). Fluorescein angiogram (FFA) (Retinal Camera, TRC-50DX, Topcon Corporation, Tokyo, Japan) revealed early hyperfluorescence of telangiectatic bulbs in the temporal regions, and hypofluorescence consistent with the subretinal exudates in the left eye. Optical coherence tomography (OCT) (CirrusHDOCT 5000, Cail Zeiss Meditec, Inc, Clifornia USA) showed macular edema in his left eye with a foveal thickness of $802 \mu \mathrm{m}$. A diagnosis of adult-onset Coats' disease was made. Examination of his right eye was unremarkable. Intraocular injection of an anti-VEGF drug (Conbercept) was performed. Before injection, the aqueous humor was removed and the levels of VEGF and inflammatory cytokines were measured. The result showed a normal VEGF level and increased Interleukin (IL) -8 (Table 1). At 1 week after the first injection, the macular edema was alleviated (Fig. 2b), with an increased BCVA of 0.5. However, after 1 month, BCVA decreased again with recurrent macular edema (Fig. 2c). A second injection of anti-VEGF was administrated and again the aqueous humor was acquired before injection. The level of VEGF was low and the level of IL-8 and VACM in the aqueous humor had increased (Table 1). Macular edema remained at this time, and laser coagulation was given at 3 weeks after injection. At 1 month after the second injection, BCVA dropped to 0.3. In consideration of the aqueous humor cytokine concentrations, treatment switched to intravitreal dexamethasone implant $\left(\right.$ Ozurdex $\left.^{\odot}\right)$ (Allergan, Inc., Irvine, Calif., USA). Then the macular edema decreased dramatically (Fig. 2d) and BCVA increased to 0.6 at 2 weeks after injection. No further recurrence of macular edema was noted during 12 weeks of follow-up. Intraocular pressure was elevated at 10 weeks after injection $(29 \mathrm{mmHg})$. After cartiolol hydrochloride eye drops were administrated 2

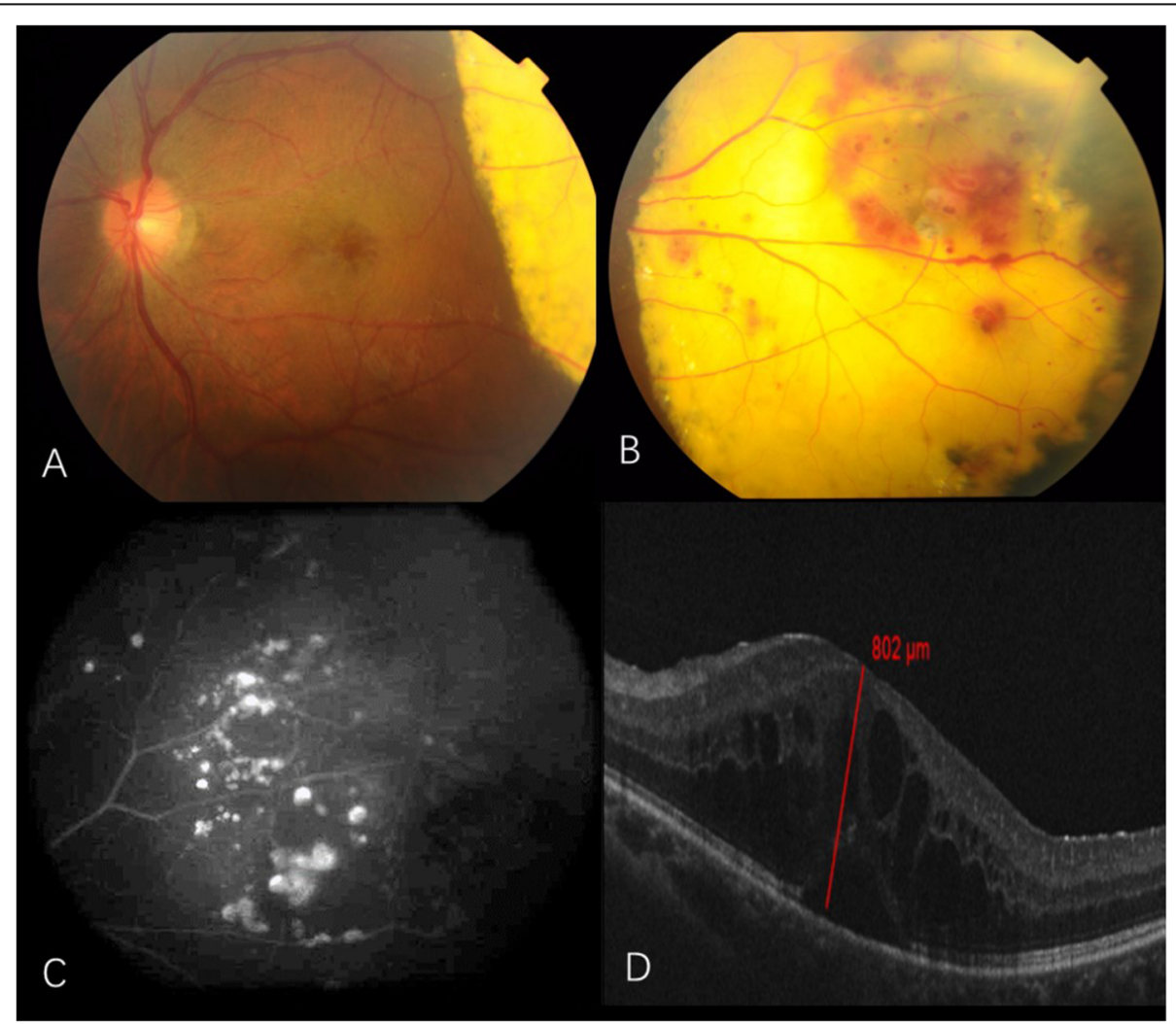

Fig. 1 Case 1: $\mathbf{a}$ and $\mathbf{b}$ Color photograph showing dense exudation with retinal telangiectasias and aneurysmal dilatations in the temporal retina; c Fluorescein angiography showing characteristic light bulb aneurysms and capillary nonperfusion areas seen temporally; $\mathbf{d}$ Optical coherence tomography showing macular edema with a foveal thickness of $802 \mu \mathrm{m}$ 


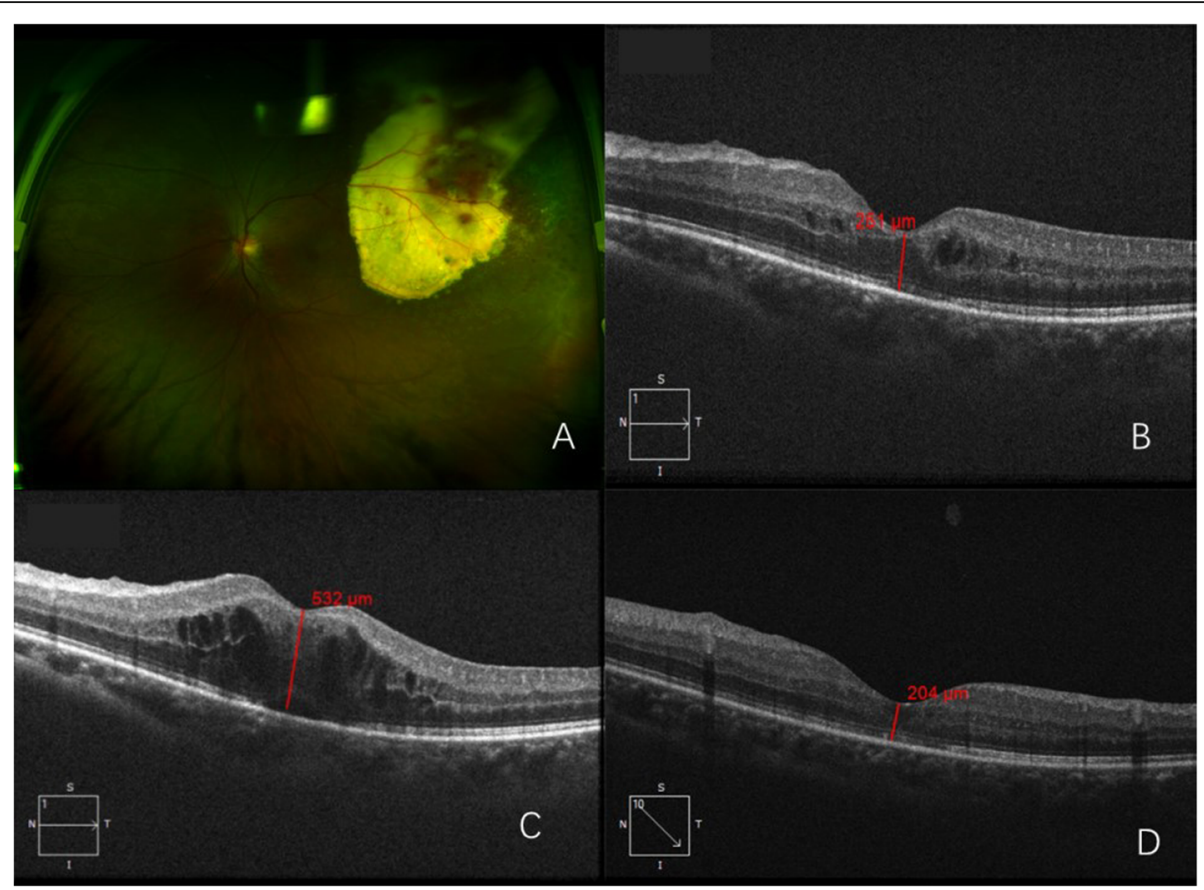

Fig. 2 Case 1: a Ultra-wide field pseudo-color photograph (Daytona, P200T, OPTOS PLC, Dunfermiline, UK) showing exudation with retinal telangiectasias, aneurysmal dilatations and temporally dated laser spots; $\mathbf{b}$ Optical coherence tomography (OCT) showing decreased macular edema after intraocular Conbercept injection; c 4th week after the intravitreal Conbercept injection, OCT showing macular edema recurrence; $\mathbf{d}$ 6th week after the intravitreal dexamethasone implant injection, OCT shows decreased macular thickness. The retina thickness at the central fovea is $204 \mu \mathrm{m}$

times a day, the IOP reduced to a normal level. No other adverse effect of the steroid was observed during the course of treatment and follow-up.

\section{Case 2}

A 40-year-old man with 15 months history of macular edema related to Coats' disease and progressive vison loss in his left eye within 4 weeks was admitted. BCVA was 1.0 in his right eye and 0.2 in his left eye. Subretinal lipid exudates in the temporal and nasal retina with local vascular telangiectasis was observed in the left eye (Fig. 4a). A fluorescein angiogram (FFA) revealed hyperfluorescence of the telangiectatic bulbs, and leaking vessels in the temporal and nasal regions (Fig. 3a, b). OCT showed increased macular thickness, cystoid edema and mild epiretinal membrane. (Fig. 3c). A diagnosis of adult-onset Coats' disease was

Table 1 Aqueous humor cytokine concentrations and macular thickness

\begin{tabular}{lllll}
\hline cytokines & Case 1 $^{1 \text { st }}$ & Case 1 $^{\text {2nd }}$ & Case 2 & Normal range \\
\hline VEGF $(\mathrm{pg} / \mathrm{mL})$ & 26.0 & 1.9 & 18.6 & $0 \sim 40$ \\
IL-6 $(\mathrm{pg} / \mathrm{mL})$ & 36.4 & 46.0 & 160.1 & $1.0 \sim 50$ \\
IL-8 $(\mathrm{pg} / \mathrm{mL})$ & 38.7 & 35.4 & 39.4 & $0 \sim 20$ \\
VCAM $(\mathrm{pg} / \mathrm{mL})$ & 810.5 & 1626.6 & 1271.3 & $200 \sim 1000$
\end{tabular}

VEGF Vascular endothelial growth factor; IL Interleukin; VCAM vascular cell adhesion molecule made. Before he presented to our clinic, he had received 11 intraocular injections of an anti-VEGF drug (Conbercept) and five laser treatments. Initially, he responded well to anti-VEGF drugs; however, there was no improvement after the final injection (Fig. 3d). When he presented to our clinic, he was treated with another anti-VEGF drug via intraocular injection (Aflibercept) and aqueous humor was obtained before the injection. Aqueous humor cytokine concentrations detection showed that VEGF was lower than normal, while VACM, IL-6 and IL-8 were at a very high level (Table 1). At 1 week after injection, macular thickness decreased from $727 \mu \mathrm{m}$ to $459 \mu \mathrm{m}$ (Fig. 3d, e); however, it increased to $490 \mu \mathrm{m}$ again after 2 weeks (Fig. 3f). Although epiretinal membrane may be also related to the recurrence of macular edema and may influence the cytokine levels. In consideration of the high inflammatory factors and lower VEGF level, an intraocular steroid $\left(\right.$ Ozurdex $\left.^{\circ}\right)$ injection was administrated. At 10 days after injection, the macular thickness dropped to $255 \mu \mathrm{m}$ and BCVA increased to 0.5 (Fig. 4b, c). During 3 months of follow-up, visual acuity was stable without any further recurrence of edema.

\section{Discussion and conclusions}

Coats' disease is classified into 5 stages depending on the severity at presentation: stage 1, telangiectasia only; 


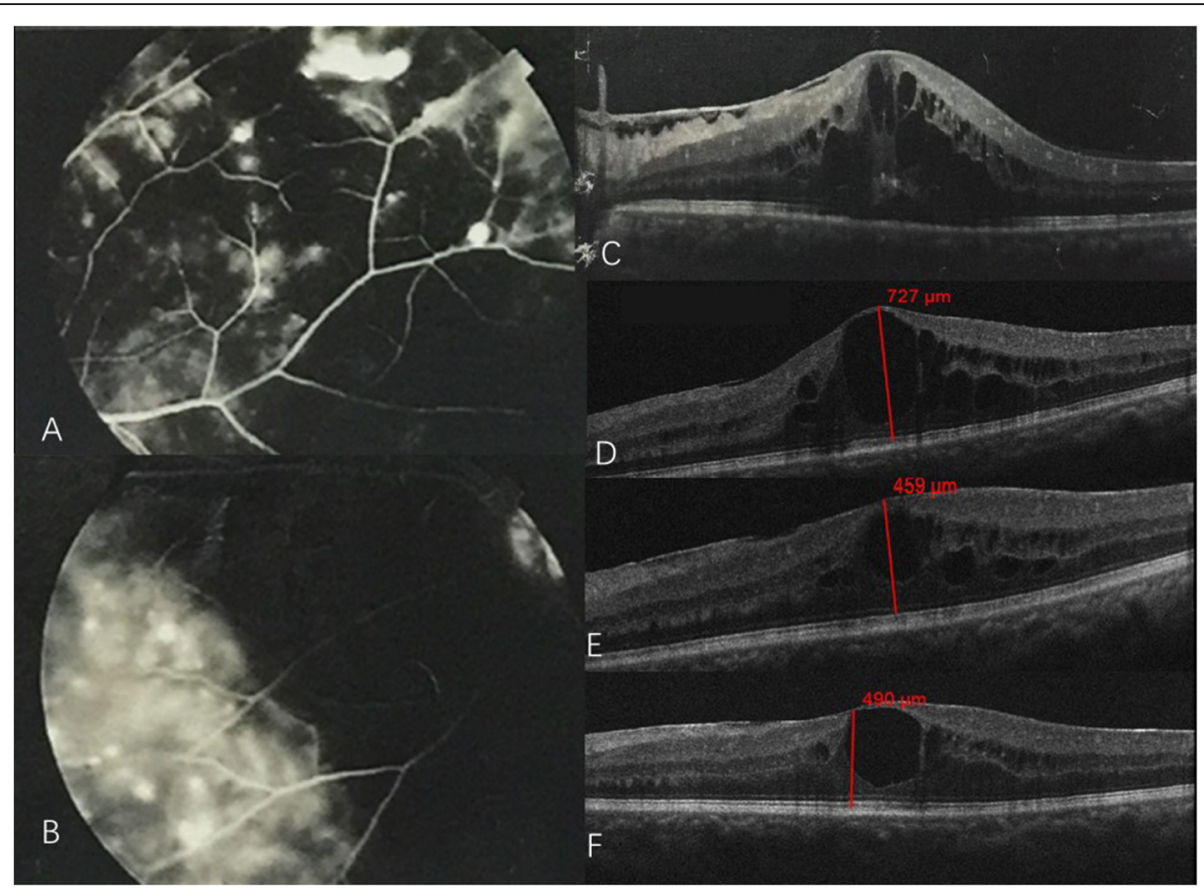

Fig. 3 a and $\mathbf{b}$ Fluorescein angiography showing characteristic light bulb aneurysms, capillary nonperfusion areas and leakage from telangiectatic vessels seen temporally and nasally; c OCT showing severe macular edema before treatment; (d) OCT showing refractory macular edema after 11 treatments of anti-VEGF drug intraocular injection; e 1st week after the intravitreal Aflibercept injection, OCT showing decreased macular thickness; $\mathbf{f} 4$ th week after the intravitreal injection, OCT showing slightly increased macular thickness

stage 2, telangiectasia and exudation (2A, extrafoveal exudation; $2 \mathrm{~B}$, foveal exudation); stage 3 , exudative retinal detachment (3A, subtotal; 3B, total); stage 4 , total detachment and secondary glaucoma; and stage 5 , advanced end-stage disease [5]. The two cases presented were both stage $2 \mathrm{~A}$ with extrafoveal exudation; macular edema was the main cause of visual impairment.
While the exact etiology of Coats' disease remains uncertain, elevated VEGF is found in Coats disease [1], which indicates that VEGF may play an important role in Coats' disease. Reports have shown that treating the persistent macular edema with anti-VEGF drugs lead to a rapid reduction of the intraretinal liquid as well as improvement of visual acuity $[6,7]$.

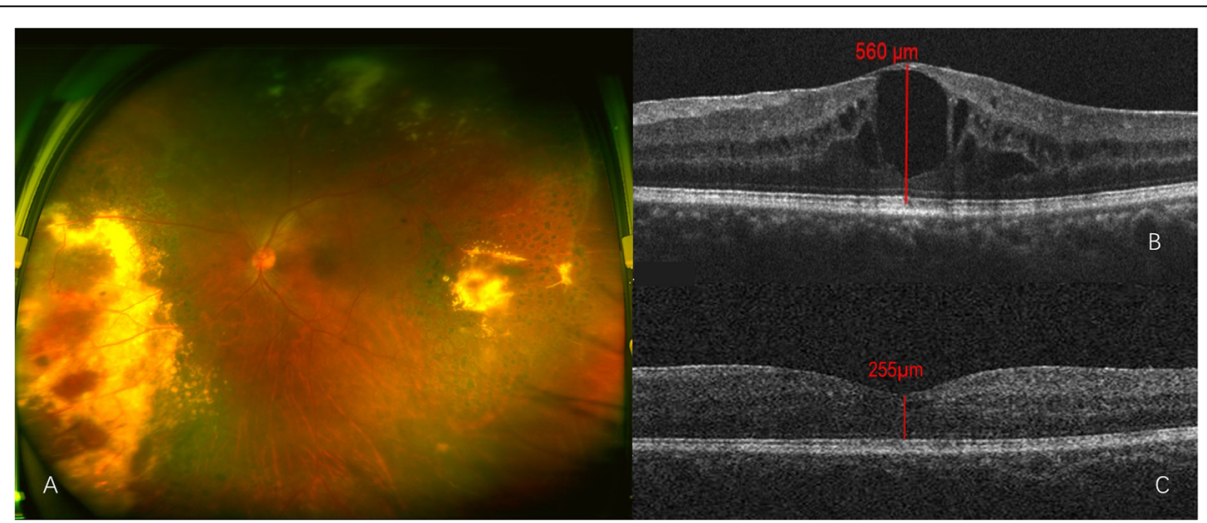

Fig. 4 a Ultra-wide field pseudo-color photograph showing subretinal lipid exudates in temporal and nasal retina with local vascular telangiectasis and dated laser spots; $\mathbf{b}$ OCT showing retina thickness at the central fovea is $560 \mu \mathrm{m}$ before intravitreal dexamethasone implant injection; c 12th week after the intravitreal dexamethasone implant injection, OCT showing decreased macular thickness. The retina thickness at the central fovea is $255 \mu \mathrm{m}$ 
Adult-onset Coats' disease manifests in a limited area of involvement and seems to advance at a slower rate than it does in children, with most patients reaching a final stable level of visual acuity [8]. Recently, Jing Feng et al. investigated the differences in aqueous concentrations of cytokines in pediatric and adult patients with Coats' disease; they found that the increasing severity of Coats' disease is significantly associated with intraocular VEGF concentration in pediatric patients, and that IL-6 may be involved with the inflammatory process in adult patients with Coats'disease [2]. Increased IL-6 concentration was found in one of our cases. Inflammatory cytokines IL-8 and VCAM increased more remarkably than IL- 6 in our cases. IL- 8 bears the primary responsibility for the recruitment of monocytes and neutrophils, the signature cells of acute inflammatory response [9]. VCAM is crucial for leukocyte recruitment and extravasation during the inflammation process [10]. MCP-1 was also reported higher in pediatric patients with Coats' disease than in the control group. Although it is still unknown that MCP-1 level in patients with adult-onset patients, it showed a strongly positive correlation with the extent of retinal exudation [11]. Jun et al. reported an adult patient with Coats' disease, whose symptom did not improve after intravitreal bevacizumab, while after intravitreal triamcinolone acetonide, the patient showed an obvious improvement in visual acuity and macular edema [12]. Recently, the efficacy of dexamethasone intravitreal implant in the management of adult-onset Coats' disease has been reported [13]. Since anti-VEGF and anti-inflammatory treatments were both reported to be effective, how to choose the proper treatment remains a question. In our cases, a low VEGF level and a high level of high inflammatory cytokines may explain the poor result of anti-VEGF therapy and the better result of steroid treatment.

The two cases highlight the precise guiding role of aqueous humor cytokine concentrations for the treatment of refractory macular edema in adult-onset Coats' disease. To the best of our knowledge, little information has been published regarding clinical response and aqueous humor cytokine concentrations in adult-onset Coats' disease following treatment with anti-VEGF or Ozurdex ${ }^{\oplus}$.

\section{Abbreviations}

VEGF: Vascular endothelial growth factor; IL: Interleukin; VCAM: Vascular cell adhesin molecule; IOP: Intraocular pressure; OCT: Optical coherence tomography

\section{Acknowledgements}

None relevant.

\section{Authors' contributions}

YW and HF have equally contributed to paper preparation and writing. YT and $\mathrm{WH}$ have critically reviewed the paper. All authors read and approved the final manuscript.

\section{Funding}

This study was supported by Technology Innovation Program of Hunan Province (Grant No. 2017SK50901), Scientific Research Program of Beijing Municipal Commission of Education(KM202010025020) and the Beijing Chaoyang 1351 talent training program (No. CYXX-2017-21). The funding organizations had no role in the design or conduct of this research.

Availability of data and materials

Not applicable.

Ethics approval and consent to participate

Not applicable.

\section{Consent for publication}

Consent for the publication of this case report, any additional related information and images was taken from the patient involved in the study. The informed consent obtained from the patient had been in writing.

\section{Competing interests}

The authors declare that they have no competing interests.

\section{Author details}

${ }^{1}$ Dalian He Eye Hospital, Dalian, China. ${ }^{2}$ Shanghai Aier Eye Hospital, Shanghai, China. ${ }^{3}$ Department of Ophthalmology, He Eye Hospital, He University, No.128, North Huanghe Street, Shenyang 110034, China. ${ }^{4}$ Department of Ophthalmology, Beijing Chaoyang Hospital, Capital Medical University, No. 8, South Road of Worker's Stadium, Chaoyang District, Beijing 100020, China.

Received: 13 January 2020 Accepted: 18 May 2020

Published online: 30 June 2020

\section{References}

1. Sun Y, Jain A, Moshfeghi DM. Elevated vascular endothelial growth factor levels in coats disease: rapid response to pegaptanib sodium. Graefes Arch Clin Exp Ophthalmol. 2007;245(9):1387-8.

2. Feng J, Zheng X, Li B, Jiang Y. Differences in aqueous concentrations of cytokines in paediatric and adult patients with Coats' disease. Acta Ophthalmol. 2017;95(6):608-12.

3. Luckie AP, Hamilton AM. Adult Coats' disease in branch retinal vein occlusion. Aust N Z J Ophthalmol. 1994;22(3):203-6.

4. Raoof N, Quhill F. Successful use of intravitreal bevacizumab and pascal laser photocoagulation in the management of adult Coats' disease. Middle East Afr J Ophthalmol. 2013;20(3):256-8.

5. Shields JA, Shields CL, Honavar SG, et al. Classification and management of coats disease: the 2000 proctor lecture. Am J Ophthalmol. 2001;131(5):572-83.

6. Strueven V, Schalenbourg A, Wolfensberger TJ. Multiple arterial macroaneurysms in acquired Coats' disease in an adult - a case report. Klin Monatsbl Augenheilkd. 2015:232(4):583-4.

7. Sen M, Shields CL, Honavar SG, Shields JA. Coats disease: an overview of classification, management and outcomes. Indian J Ophthalmol. 2019;67(6):763-71.

8. Smithen LM, Brown GC, Brucker AJ, et al. Coats' disease diagnosed in adulthood. Ophthalmology. 2005;112(6):1072-8.

9. Apostolakis S, Vogiatzi K, Amanatidou V, Spandidos DA. Interleukin 8 and cardiovascular disease. Cardiovasc Res. 2009;84(3):353-60.

10. $\mathrm{Xu} \mathrm{H}$, Forrester JV, Liversidge J, Crane IJ. Leukocyte trafficking in experimental autoimmune uveitis: breakdown of blood-retinal barrier and upregulation of cellular adhesion molecules. Invest Ophthalmol Vis Sci. 2003:44(1):226-34

11. Zhang J, Jiang C, Ruan L, Huang X. Associations of cytokine concentrations in aqueous humour with retinal vascular abnormalities and exudation in Coats' disease. Acta Ophthalmol. 2019;97(3):319-24.

12. Jun JH, Kim YC, Kim KS. Resolution of severe macular edema in adult coats' disease with intravitreal triamcinolone and bevacizumab injection. Korean J Ophthalmol. 2008;22(3):190-3.

13. Kumar K, Raj P, Chandnani N, Agarwal A. Intravitreal dexamethasone implant with retinal photocoagulation for adult-onset Coats' disease. Int Ophthalmol. 2019;39(2):465-70.

\section{Publisher's Note}

Springer Nature remains neutral with regard to jurisdictional claims in published maps and institutional affiliations. 